\title{
Factor Structure of the Social Communication Questionnaire in Preschool Aged Autistic Children
}

\author{
Rachel Grove $\mathbb{1}^{1,2} \cdot$ Jessica Paynter ${ }^{3} \cdot$ Annette Joosten $^{4,5} \cdot$ Giacomo Vivanti $^{6,7} \cdot$ Cheryl Dissanayake $^{6}$. \\ Valsamma Eapen ${ }^{8}$
}

(c) Springer Science+Business Media, LLC, part of Springer Nature 2019

\begin{abstract}
Objectives The Social Communication Questionnaire (SCQ) is a common screening tool for autism spectrum disorder. Given its wide use, as well as recent changes to diagnostic criteria for autism, there is a need to evaluate whether the SCQ maps onto the social communication and interaction, and repetitive behaviour and restricted interests domains specified in the revised criteria in DSM-5.

Methods Participants included 294 preschool aged autistic children. Confirmatory factor analysis was implemented to evaluate the factor structure of the SCQ.

Results None of the models evaluated provided an adequate fit to the data. However, the best fitting model mapped onto the previous DSM-IV diagnostic criteria for autism.

Conclusions The SCQ does not align with the new DSM-5 domains. More research is needed to evaluate the factor structure of the SCQ in different populations of autistic children in order to determine its current validity as a screening tool.
\end{abstract}

Keywords Autism spectrum disorder $\cdot$ Social communication questionnaire $\cdot$ Factor analysis $\cdot$ Preschool children $\cdot$ DSM-5

Autism spectrum disorder is a neurodevelopmental condition with a prevalence of 1 in 59 in the US (Centers for

Rachel Grove

rachel.grove@uts.edu.au

1 Graduate School of Health, University of Technology Sydney, Sydney, NSW, Australia

2 School of Psychiatry, University of New South Wales, Sydney, NSW, Australia

3 School of Applied Psychology, Griffith University, Gold Coast, QLD, Australia

4 School of Occupational Therapy and Social Work, Faculty of Health Sciences, Perth, WA, Australia

5 Autism Association of Western Australia, Curtin University, Perth, WA, Australia

6 Olga Tennison Autism Research Centre, School of Psychology and Public Health, La Trobe University, Melbourne, VIC, Australia

7 AJ Drexel Autism Institute, Drexel University, Philadelphia, PA, USA

8 School of Psychiatry, University of New South Wales, Sydney, NSW, Australia
Disease Control and Prevention 2014) and between 1.5 and $2.5 \%$ of children in Australia (Randall et al. 2016). The assessment and diagnosis of autism has undergone some substantial changes over the last decade. Traditionally, autism spectrum disorders have been understood as consisting of a triad of impairments in social interaction, social communication and restricted and repetitive behaviours or interests (RRBIs) (Wing 1981; Wing and Gould 1979). The fourth edition of the Diagnostic and Statistical Manual of Mental Disorders (DSM-IV; American Psychiatric Association 2000) defined the diagnostic criteria for autism spectrum disorders based on this triad. However, the DSM-5 criteria for autism spectrum disorder combine the social interaction and communication difficulties into a single domain alongside the RRBIs domain, outlining two, rather than three, domains for diagnosis. The changes to the diagnostic criteria pose a challenge for the assessment and diagnosis of autism moving forward in both clinical practice and research (Vivanti et al. 2013).

The 'gold-standard' diagnostic assessments for autism include the Autism Diagnostic Interview Revised (ADI-R; Lord et al. 1994) and the Autism Diagnostic Observation Schedule (ADOS; Lord et al. 2012). However, the administration of these gold standard assessments can be time 
consuming and costly for both practitioners and parents, limiting their use, and potentially increasing the likelihood that autistic children are missed. Given the importance of early recognition and provision of support for autistic children (Myers and Johnson 2007), it is critical that diagnosis is provided as early as possible. Some authors have argued that it is therefore both important and efficient to use a two-phase approach to diagnosis (Glascoe 2005), in which a brief screening measures is followed up with a more comprehensive assessment where necessary. In addition, screening measures are often used as a proxy to verify diagnosis in research protocols (e.g. for epidemiological research and for the comparison of autistic traits across different populations) where a more comprehensive assessment is logistically not feasible. A number of screening measures have thus been developed over the last two decades to meet these needs.

The Social Communication Questionnaire (SCQ; Berument et al. 1999; Rutter et al. 2003) is one of the most commonly used screening measures in the field. It was originally titled the Autism Screening Questionnaire and was developed based on the ADI-R (Lord et al. 1994). The SCQ contains 40 items designed to evaluate children across the three DSM-IV domains of social interaction, communication and RRBIs (American Psychiatric Association 2000). While the items of the SCQ are defined based on these three diagnostic criteria, a total score above a cut off of 15 is used to indicate a probable diagnosis for autism (Rutter et al. 2003). The majority of the research on the SCQ evaluates the utility of this total score, generally indicating that it displays good sensitivity and specificity (Berument et al. 1999). While this research provides valuable information on the utility of the SCQ, the mean participant age of 23 years raises questions about its applicability to children who are typically screened for autism. There have been several studies that evaluate the utility of the recommended cut off score for the SCQ across different age groups. This research predominantly highlights that the SCQ performs better when used with an older age group, and that a lower threshold is required to effectively screen for autism in children under the age of five (Corsello et al. 2007; Rosenberg et al. 2018; Wiggins et al. 2007). However, there is also some evidence for the utility of this measure in children under four years of age (Allen et al. 2007; Charman et al. 2016; Eapen et al. 2013; Lee et al. 2007; Paynter et al. 2013).

There have been three factor analytic studies to date evaluating the structure of the SCQ and whether it is more effective to use a total score or a number of underlying subscales. Berument et al. (1999) identified four factors they labelled social interaction, communication, abnormal language, and stereotyped behaviour (Berument et al.
1999). However, this original paper did not evaluate the fit of the SCQ to the DSM-IV model. Wei et al. (2014) used confirmatory factor analysis to evaluate the four factors outlined by Berument et al. (1999) and used item response theory (IRT) to evaluate the SCQ subscales. They found sufficient unidimensionality for the language and communication scale (two factors) and for the RRBI scale, but a poor fit for social interaction. However, this study evaluated each factor individually, therefore limiting the ability to determine the relationship between the four factors and an overall estimation of the internal structure of the SCQ. Magyar et al. (2012) also evaluated the SCQ using factor analytic techniques and found support for a two-factor solution in a group of individuals with Down syndrome alone, or with a comorbid diagnosis of autism. There is a need for a comprehensive assessment of the factor structure of the SCQ in order to determine the most appropriate scale to use when screening for autism in both research and clinical practice. It is also important to determine how diagnostic screening measures such as the SCQ, developed according to the three DSM-IV domains map onto the current two-factor conceptualisation outlined in DSM-5.

The aim in the present study was to determine whether a unidimensional (total score), two factor (DSM-5), three factor (DSM-IV) or four-factor (Berument et al. 1999) model provides the best conceptualisation of the SCQ in preschool aged autistic children.

\section{Method}

\section{Participants}

The sample consisted of 294 children $\quad$ (Male $=239$, Female $=55$ ) enrolled at four autism specific early intervention centres, with a mean age of 3.9 years (Range $=$ $1.7-6.1 ; \mathrm{SD}=0.8$ ). The sample comprised 163 non-verbal/ minimally verbal children and 131 verbal children, as determined on the responses provided to item 1 (i.e. talks in phrases) of the SCQ.

\section{Procedure}

All children received early intervention within a group based early learning and care setting. In order to enrol at the autism specific early intervention centres, participants need to have a diagnosis of autism spectrum disorder, with diagnosis confirmed for all participants by a practitioner (e.g. paediatrician, multidisciplinary team). Primary caregivers completed the SCQ as part of the intake process into the early intervention centres. 


\section{Measures}

\section{Social communication questionnaire}

Parents completed the 40-item SCQ (Rutter et al. 2003), designed to evaluate the presence of symptoms of autism including social interaction, communication and restricted and repetitive behaviours and interests. Items are answered on a dichotomous (yes/no) scale. The items are scored on either a 'lifetime' or 'current' form. The current form was used in this study. Item 1 is not scored, but evaluates the child's level of language. If the child does not have enough language to determine the presence of abnormalities, the first six items are not completed. A total score is computed, ranging from zero to 39 for verbal children and from zero to 33 for non-verbal/minimally verbal children. There were 163 non-verbal/minimally verbal children and 131 verbal children in the sample. Higher scores are indicative of greater endorsement of autistic traits.

\section{Data Analyses}

The frequency of endorsement of each item of the SCQ across the two groups was calculated to determine the distribution of scores. Confirmatory factor analysis (CFA) was implemented using Mplus version 7 (Muthén and Muthén 1998-2012). Four models were included to evaluate the relative fit of a one factor (39 item total score), two-factor (36 item DSM-5), three-factor (36 item DSM-IV) and fourfactor (39 item Berument et al. 1999) solution to the data. In order to account for the impact of language on scores on the SCQ, the language ability of the child (verbal versus nonverbal/minimally verbal using the dichotomous item 1) was included as a covariate in all models.

The weighted least square mean and variance adjusted estimator (WLSMV) was used in all the analyses. Model fit was evaluated using the root mean square error of approximation (RMSEA; Steiger and Lind 1980), comparative fit index (CFI; Bentler 1987) and the Tucker-Lewis index (TLI; Tucker and Lewis 1973). TLI and CFI scores above 0.90 are indicative of a good model fit (Brown 2006; $\mathrm{Hu}$ and Bentler 1999). RMSEA scores are recommended to be below 0.08 for good model fit and 0.05 for an excellent fit to the data (Browne and Cudeck 1993).

\section{Results}

The proportion of endorsement for each item of the SCQ for the non-verbal/minimally verbal and the verbal groups are given in Figs. 1-3. Scores ranged from a 12\% (Item 30) to 94\% (Item 39) frequency of endorsement.

The gender distribution of the sample and total scores on the SCQ for the non-verbal/minimally verbal and verbal participants are provided in Table 1 . The verbal children were significantly older than the non-verbal/minimally

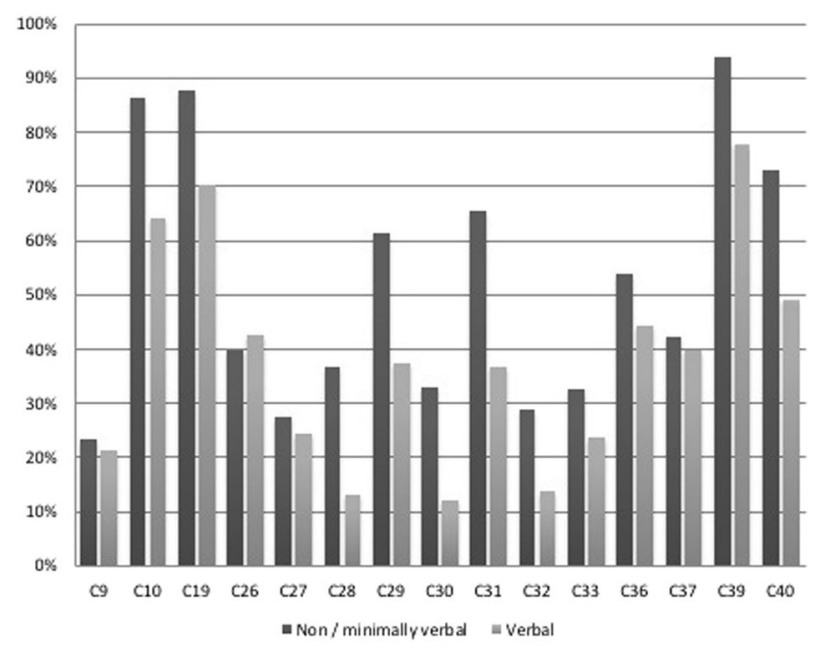

Fig. 2 Frequency of endorsement of the communication items of the SCQ
Fig. 1 Frequency of endorsement of the social interaction items of the SCQ

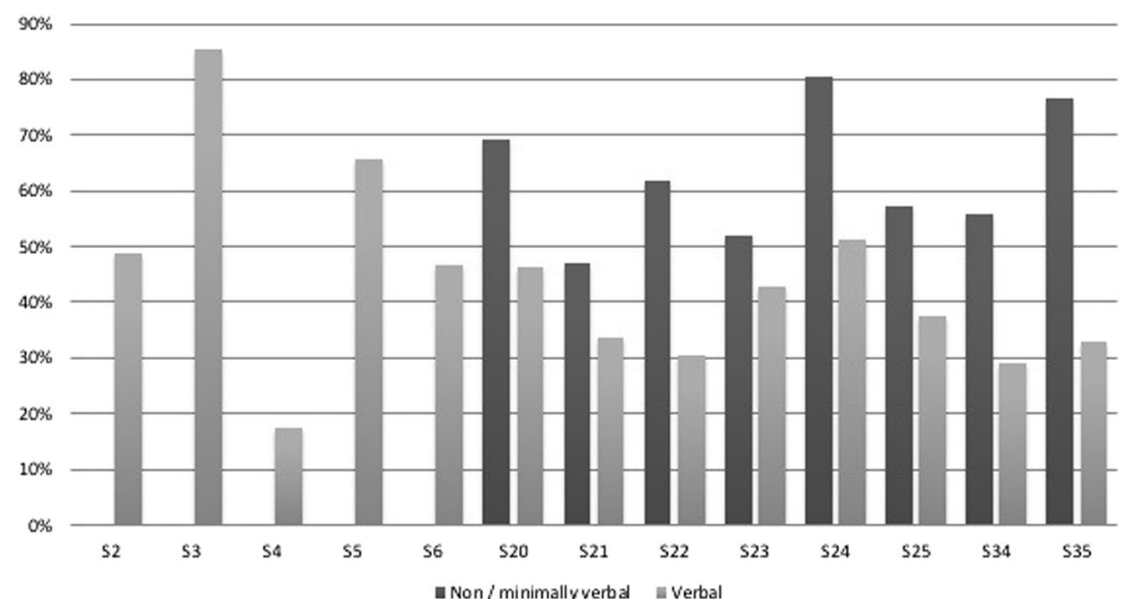




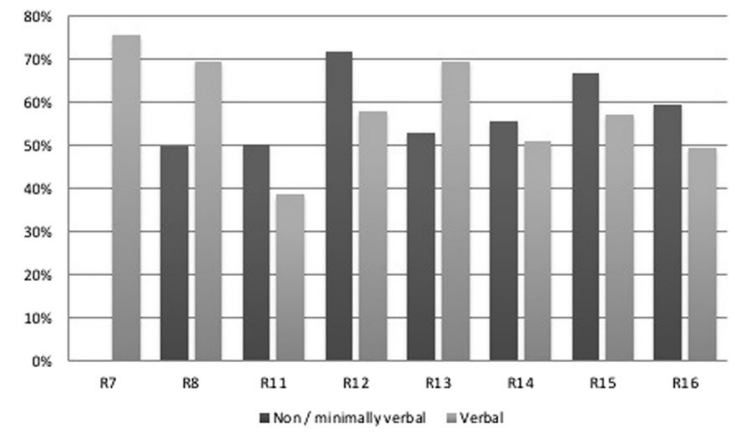

Fig. 3 Frequency of endorsement of the restricted and repetitive behaviour items of the SCQ

Table 1 Demographic information

\begin{tabular}{lll}
\hline & Non-verbal $(n=163)$ & Verbal $(n=131)$ \\
\hline \multirow{2}{*}{ Females } & Freq & Freq \\
Males & 33 & 22 \\
& 130 & 109 \\
Age & Mean (SD) & Mean (SD) \\
Total SCQ score & $3.7(0.8)$ & $4.1^{* *}(0.7)$ \\
$* * p<0.01$ & $18.2(5.5)$ & $17.4(6.7)$ \\
\hline
\end{tabular}

$* * p<0.01$

verbal group. There was no difference between total scores on the SCQ for non-verbal/minimally verbal and verbal participants.

Results of the CFA are presented in Table 2. There were minimal differences between the models evaluated. While RMSEA scores were below the recommended cut-off scores in all models, the CFI and TLI did not reach the threshold. The one-factor model provided the worst fit to the data overall. The four-factor model appeared to provide the best fit overall. However, the correlation between the language and communication factors in these models was $>0.9$, indicating that these two factors are not unique. The threefactor DSM-IV model provided the best fit to the data overall. However, while the RMSEA values were adequate across models, the CFI and TLI for all models were below the recommended cut-off scores.

Within each of the four models there were a number of items that did not contain loadings greater than the recommended threshold of $>0.32$ (Costello and Osborne 2005). There were six items that did not load onto any factor across the DSM-IV and DSM-5 models. Items 3 to 6 (evaluating communication on the SCQ) and items 8 to 13 (evaluating RRBIs on the SCQ) were shown to have factor loadings under this accepted threshold. In addition, Item 2 : Do you have a to and fro conversation with him/her that involves taking turns or building on what you have said? and Item 3: Does he/she ever use odd phrases or say the same thing over and over in almost exactly the same way? were shown to cross load on the RRBI factor. Item 8: Does he/she ever have things that he/she seems to have to do in a particular way or order or rituals that he/she insists you go through?, Item 12: Does he/she ever seem to be more interested in parts of a toy or an object rather than using the object as it was intended? and Item 13: Does he/she ever have any special interests that are unusual in their intensity but otherwise appropriate for his/her age and peer group? were also shown to cross load on the social and communication factors. This finding, coupled with the fit indices falling below the recommended thresholds indicates that none of these models described the data adequately.

\section{Discussion}

The primary aim in the current study was to evaluate the factor structure of the SCQ within a sample of preschool aged autistic children. Results indicated that the unidimensional model provided the worst fit to the data. This highlights that the use of a total score is not appropriate in this sample. Given that the use of a total score is currently recommended in the SCQ manual, and that it is widely reported in this format, our findings have significant implications for research and clinical practice. This is consistent with previous research that shows that the use of a total cut off score in isolation may not be the most effective way of screening for autism (Charman et al. 2016).

While the four-factor model outlined by Berument et al. (1999) showed slightly higher fit indices, there was a large correlation between the language and communication factors, indicating that this model did not fit the data adequately. This is interesting given the results presented by Wei et al. (2014) indicating that each of these four factors could be conceptualised on a dimensional scale. The current study evaluated the fit of this model by including all four factors within the same analysis, as opposed to individually as in the original study. This may account for the differing results between the two papers.

There was also evidence that the DSM-5 model did not provide the best fit to the data as, within the current sample, the SCQ did not align with the new DSM-5 domains. With the changes to the DSM-5, it is important that screening tools fit the current diagnostic criteria for a disorder in order to correctly identify those who require additional assessment. Given that the SCQ does not map well onto the underlying constructs outlined by DSM-5, it may limit its potential to identify those individuals likely to receive a diagnosis of autism spectrum disorder. This has significant implications for the current use of the SCQ as a screening tool and as a proxy of diagnosis in both research and clinical settings. The SCQ has not been revised to reflect the new 
Table 2 Fit indices and model comparisons for the social communication questionnaire

\begin{tabular}{llllll}
\hline Model & Description & Fit indices & & \\
\cline { 4 - 6 } & & RMSEA & CFI & TLI & Chi square \\
\hline 1 & One factor model $(n=294)$ & 0.052 & 0.757 & 0.744 & $1329.93^{* *}$ \\
2 & Four factor Berument et al. model $(n=294)$ & 0.049 & 0.784 & 0.770 & $1255.01^{* *}$ \\
3 & Two factor DSM-5 model $(n=294)$ & 0.051 & 0.797 & 0.784 & $1107.44^{* *}$ \\
4 & Three factor DSM-IV model $(n=294)$ & 0.050 & 0.806 & 0.793 & $1082.56^{* *}$ \\
\hline
\end{tabular}

$* * p<0.01$
DSM-5 domains. It is important that further research evaluating the use of the SCQ in the context of the change in the DSM 5 criteria, as well as the sensitivity and specificity of the SCQ across different populations and settings be conducted.

A three-factor model representing the social interaction, communication and RRBI subscales outlined in DSM-IV provided the best fit to the data. Although this model provided the best fit overall, there were still problems with the psychometric properties of the SCQ, as a number of items accounted for a minimal proportion of the variance in the model. There were also six items that did not load onto any factor across both the DSM-IV and DSM-5 models, as well as items that cross loaded onto additional factors. This indicates that there are a number of items on the SCQ that may not map onto the diagnostic criteria of autism effectively. This may be due to the large range in the endorsement of items (i.e. from 12 to $94 \%$ ), indicating that there is substantial variation in the response rates across the SCQ. In addition, the dichotomous scoring on the SCQ reduces the variance or spread that can be obtained within each item. Moreover, a number of the items are not answered depending on the verbal ability of the child. This may have an additional impact on the variance within the model. While not within the scope of the current study, further research evaluating the individual items of the SCQ and their utility is warranted.

The findings of this study suggest the commonly used single score of the SCQ does not reflect a unitary construct and thus caution should be used in its interpretation. Further, the SCQ does not appear to map well onto the revised two-domain structure of the DSM-5. This suggests that the SCQ may not be appropriate for use as either as a screening tool or to verify diagnosis. This is consistent with previous research indicating that the accuracy of the SCQ as a screening tool is moderate, and that these measures should not be used alone to determine whether an individual should be referred for a full developmental assessment (Charman et al. 2016). Further research evaluating the SCQ has also indicated that the sensitivity and specificity of the measure is lower than first reported (Barnard-Brak et al. 2016). In addition, the SCQ does not include any items evaluating sensitivity to sensory input or sensory interests, which now form part of the revised diagnostic criteria for autism. This highlights the need to develop comprehensive screening tools that align more closely with the new DSM-5 criteria for autism.

Furthermore, this study aligns with recent research indicating that the SCQ may not be appropriate for use with children under the age of four (Chesnut et al. 2016; Marvin et al. 2017). Given that other screening tools such as the Modified Checklist for Autism in Toddlers (Robins et al. 2014) have an upper age limit of 30 months, there is a need for the development of appropriate screening tools for children aged between 30 and 48 months.

\section{Limitations and Future Research}

There were a number of limitations in the current study. First, the inclusion of an older age group would have been beneficial in order to compare the internal structure of the SCQ in a cohort similar to that used in other studies. However, given that the SCQ has been shown to have utility in preschool aged children, it is important to evaluate the SCQ in this younger sample. It would also be beneficial to include other diagnostic measures of autism including the ADI-R and ADOS, in order to allow for comparisons of the SCQ and 'gold-standard' diagnostic assessment tools. As in previous studies evaluating autism, the sample comprised a larger proportion of males than females. Future research should also evaluate whether the SCQ is equally appropriate for use in screening for autism in both male and female populations. The changes to the diagnostic criteria in DSM5 have brought new challenges and opportunities for research into autism. The next challenge is to develop appropriate screening measures that map well onto DSM-5, and are beneficial for both research and clinical practice.

Acknowledgements This project was funded by the Department of Social Services. The first author was supported by the Cooperative Research Centre for Living with Autism (Autism CRC), established and supported under the Commonwealth Government's Cooperative Research Centres programme. The authors would like to thank all staff at the Autism Specific Early Learning and Care Centres involved in this study. We also sincerely thank the children and families who participated in this research.

Authors' contributions RG: designed the study, completed all data analyses, and drafted the manuscript. JP: collaborated with the design 
of the study, provided data, and assisted with the drafting of the manuscript. AJ: collaborated with the design of the study, provided data, and assisted with the drafting of the manuscript. GV: collaborated with the design of the study, provided data, and assisted with the drafting of the manuscript. CD: collaborated with the design of the study, and assisted with the drafting of the manuscript. VE: designed the study and assisted with the drafting of the manuscript.

\section{Compliance with ethical standards}

Conflict of interest The authors declare that they have no conflict of interest.

Ethical approval Ethical approval to conduct this study was obtained via the University of New South Wales Human Research Ethics Committee prior to the study commencing, and appropriate ethical guidelines were followed. Informed consent was obtained from all parents before their child was enrolled in the study.

Publisher's note: Springer Nature remains neutral with regard to jurisdictional claims in published maps and institutional affiliations.

\section{References}

Allen, C. W., Silove, N., Williams, K., \& Hutchins, P. (2007). Validity of the social communication questionnaire in assessing risk of autism in preschool children with developmental problems. Journal of Autism and Developmental Disorders, 37(7), 1272-1278. https://doi.org/10.1007/s10803-006-0279-7.

American Psychiatric Association. (2000). Diagnostic and Statistical Manual of Mental Disorders DSM-IV-TR. 4th ed. Washington DC: American Psychiatric Publishing Inc.

Barnard-Brak, L., Brewer, A., Chesnut, S., Richman, D., \& Schaeffer, A. M. (2016). The sensitivity and specificity of the social communication questionnaire for autism spectrum with respect to age. Autism Research, 9(8), 838-845. https://doi.org/10.1002/aur.1584.

Bentler, P. M. (1987). Comparative fit indices in structural models. Psychological Bulletin, 107, 238-246. https://doi.org/10.1037/ 0033-2909.88.3.588.

Berument, S. K., Rutter, M., Lord, C., Pickles, A., \& Bailey, A. (1999). Autism screening questionnaire: Diagnostic validity. British Journal of Psychiatry, 175, 444-451. https://doi.org/10. 1192/bjp.175.5.444.

Brown, T. A. (2006). Confirmatory factor analysis for applied research. New York, NY: The Guildford Press.

Browne, M. W., \& Cudeck, R. (1993). Alternate ways of assessing model fit. In K. A. Bollen \& J. S. Long (Eds), Testing structural equation models (pp. 136-162). Newbury Park, CA: Sage.

Centers for Disease Control and Prevention. (2014). Prevalence of autism spectrum disorder among children aged 8 years - autism and developmental disabilities monitoring network, 11 Sites, United States, 2010. MMWR Surveillance Summaries, 63, $1-21$.

Charman, T., Baird, G., Simonoff, E., Chandler, S., Davison-Jenkins, A., \& Sharma, A., et al. (2016). Testing two screening instruments for autism spectrum disorder in UK community child health services. Developmental Medicine and Child Neurology, 58, 369-375. https://doi.org/10.1111/dmcn.12874.

Chesnut, S. R., Wei, T., Barnard-Brak, L., \& Richman, D. M. (2016). A meta-analysis of the social communication questionnaire: Screening for autism spectrum disorder. Autism, 21(8), 920-928. https://doi.org/10.1177/1362361316660065.
Corsello, C., Hus, V., Pickles, A., Risi, S., Cook, Jr, E. H., \& Leventhal, B. L., et al. (2007). Between a ROC and a hard place: Decision making and making decisions about using the SCQ. Journal of Child Psychology and Psychiatry and Allied Disciplines, 48(9), 932-940. https://doi.org/10.1111/j.1469-7610. 2007.01762.x.

Costello, A. B., \& Osborne, J. W. (2005). Best practices in exploratory factor analysis: four recommendations for getting the most from your analysis. Practical Assessment, Research and Evaluation, 10(7), 1-9.

Eapen, V., Crncec, R., \& Walter, A. (2013). Clinical outcomes of an early intervention program for preschool children with Autism Spectrum Disorder in a community group setting. BMC Pediatrics, 13, 3. https://doi.org/10.1186/1471-2431-13-3.

Glascoe, F. P. (2005). Screening for developmental and behavioral problems. Mental Retardation and Developmental Disabilities Research Reviews, 11(3), 173-179. https://doi.org/10.1002/mrdd. 20068.

Hu, L., \& Bentler, P. M. (1999). Cutoff criterion for fit indices in covariance structure analysis: Conventional criteria versus new alternatives. Structural Equation Modeling, 6, 1-55. https://doi. org/10.1080/10705519909540118.

Lee, L. C., David, A. B., Rusyniak, J., Landa, R., \& Newschaffer, C. J. (2007). Performance of the Social Communication Questionnaire in children receiving preschool special education services. Research in Autism Spectrum Disorders, 1(2), 126-138. https:// doi.org/10.1016/j.rasd.2006.08.004.

Lord, C., Rutter, M., DiLavore, P. C., Risi, S., Gotham, K., \& Bishop, S. L. (2012). Autism diagnostic observation schedule (ADOS-2). 2nd ed. Los Angeles: Western Psychological Services.

Lord, C., Rutter, M., \& Le Couteur, A. (1994). Autism diagnostic interview-revised: A revised version of a diagnostic interview for caregivers of individuals with possible pervasive developmental disorders. Journal of Autism and Developmental Disorders, 24, 659-685. https://doi.org/10.1007/BF02172145.

Magyar, C. I., Pandolfi, V., \& Dill, C. A. (2012). An initial evaluation of the social communication questionnaire for the assessment of autism spectrum disorders in children with down syndrome. Journal of Developmental and Behavioral Pediatrics, 33(2), 134-145. https://doi.org/10.1097/DBP.0b013e318240d3d9.

Marvin, A. R., Marvin, D. J., Lipkin, P. H., \& Law, J. K. (2017), Analysis of Social Communication Questionnaire (SCQ) Screening for Children Less Than Age 4. Current Developmental Disorders Reports, 4(4), 137-144. https://doi.org/10.1007/ s40474-017-0122-1.

Muthén, L. K., \& Muthén, B. O. (1998-2012). Mplus User's Guide. 7th ed. Los Angeles, CA: Muthén \& Muthén.

Myers, S. M., \& Johnson, C. P. (2007). American academy of pediatrics council on children with disabilities. Management of children with autism spectrum disorders. Pediatrics, 120, 1162-1182.

Paynter, J., Riley, E., Beamish, W., Davies, M., \& Milford, T. (2013). The double ABCX model of family adaptation in families of a child with an autism spectrum disorder attending an Australian early intervention service. Research in Autism Spectrum Disorders, 7, 1183-1195. https://doi.org/10.1016/j.rasd.2013.07.006.

Randall, M., Brignall, A., Sciberras, E., Ihsen, E., Efron, D., \& Dissanayake, C., et al. (2016). Autism Spectrum Disorder: presentation and prevalence in a nationally representative Australian sample. Australian and New Zealand Journal of Psychiatry, 50, 243-253. https://doi.org/10.1177/0004867415595287.

Robins, D. L., Casagrande, K., Barton, M., Chen, C. M., DumontMathieu, T., \& Fein, D. (2014). Validation of the modified checklist for autism in toddlers, revised with follow-up (MCHAT-R/F). Pediatrics, 33, 37-45. 
Rosenberg, S. A., Moody, E. J., Lee, L.-C., DiGuiseppi, C., Windham, G. C., \& Wiggins, L. D., et al. (2018). Influence of family demographic factors on social communication questionnaire scores. Autism Research, 11(5), 695-706. https://doi.org/10. 1002/aur.1935.

Rutter, M. L., Bailey, A., \& Lord, C. (2003). Social Communication Questionnaire $(S C Q)$. Los Angeles, CA: Western Psychological Services.

Steiger, J. H., \& Lind, J. M. (1980). Statistically based tests for the number of common factors. Iowa City, IA: Meeting of the Psychometric Society.

Tucker, L. R., \& Lewis, C. (1973). A reliability coefficient for maximum likelihood factor analysis. Psychometrika, 15, 149-162. https://doi.org/10.1007/BF02291170.

Vivanti, G., Hudry, K., Trembath, D., Barbaro, J., Richdale, A., \& Dissanayake, C. (2013). Towards the DSM V criteria for autism: clinical, cultural and research implications. Australian Psychologist, 48(4), 258-261.
Wei, T., Chesnut, S., Barnard-Brak, L., \& Richman, D. (2014). Psychometric Analysis of the Social Communication Questionnaire Using an Item-Response Theory Framework: implications for the Use of the Lifetime and Current Forms. Journal of Psychopathology and Behavioral Assessment, 37, 1-12. https://doi.org/ 10.1007/s10862-014-9468-4.

Wiggins, L. D., Bakeman, R., Adamson, L. B., \& Robins, D. L. (2007). The Utility of the Social Communication Questionnaire in Screening for Autism in Children Referred for Early Intervention. Focus on Autism and Other Developmental Disabilities, 22, 33-38. https://doi.org/10.1177/10883576070220010401.

Wing, L. (1981). Language, social and cognitive impairments in autism and severe mental retardation. Journal of Autism and Developmental Disorders, 10, 31-44.

Wing, L., \& Gould, J. (1979). Severe impairments of social interaction and associated abnormalities in children: Epidemiology and classification. Journal of Autism and Developmental Disorders, 9 (1), 11-29. 